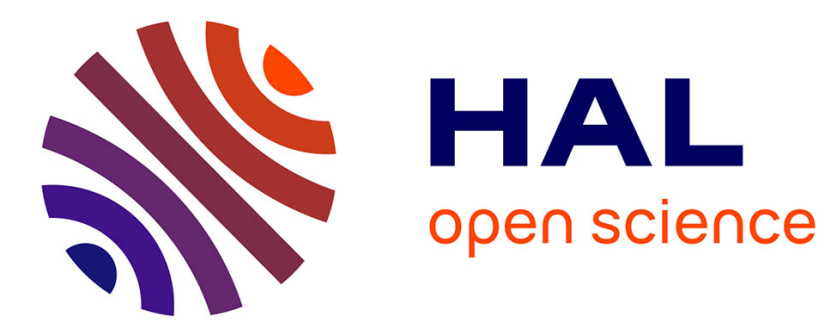

\title{
Alerte et catastrophe: le cas de la tempête de 1999, un risque scélérat
}

François Dedieu

\section{To cite this version:}

François Dedieu. Alerte et catastrophe: le cas de la tempête de 1999, un risque scélérat. Sociologie du Travail, 2009, 51, pp.379-401. 10.1016/j.soctra.2009.06.001 . hal-02054486

\section{HAL Id: hal-02054486 \\ https://hal.science/hal-02054486}

Submitted on 1 Mar 2019

HAL is a multi-disciplinary open access archive for the deposit and dissemination of scientific research documents, whether they are published or not. The documents may come from teaching and research institutions in France or abroad, or from public or private research centers.
L'archive ouverte pluridisciplinaire $\mathbf{H A L}$, est destinée au dépôt et à la diffusion de documents scientifiques de niveau recherche, publiés ou non, émanant des établissements d'enseignement et de recherche français ou étrangers, des laboratoires publics ou privés. 


\title{
Alerte et catastrophe : le cas de la tempête de 1999, un risque scélérat
}

\author{
Warnings and catastrophes: The 1999 storm, a treacherous risk \\ François Dedieu
}

Centre de sociologie des organisations, 19, rue Amélie, 75018 Paris, France

\begin{abstract}
Résumé
L'objet de cet article est de contribuer à l'explication du paradoxe récurrent des situations de catastrophe : comment expliquer qu'une catastrophe semble toujours prévue ex post et jamais évitée ex ante ? À travers l'étude de l'alerte précédant le dernier grand désastre naturel que la France ait connu — la tempête du 27 décembre 1999 - l'article se propose de rendre compte des ressorts organisationnels expliquant l'effet surprise provoqué par cette catastrophe. Il s'agit de comprendre pourquoi, alors que le phénomène a bien été annoncé et prévu par Météo-France, les acteurs de la sécurité civile se déclarèrent profondément surpris par l'ampleur de l'évènement. En proposant une analyse qualitative multiniveau du fonctionnement du système interorganisationnel de l'alerte météorologique, il est montré que, dans cet épisode, la tentative d'alerte a été transformée en un signal routinier en raison de la combinaison de facteurs structurels, contextuels et individuels. Le cas de la tempête de 1999 permet d'introduire l'idéal type d'une catégorie de risque particulière, le risque scélérat. Il s'agit d'un danger annoncé comme tel mais qui est ignoré parce qu'il semble familier.
\end{abstract}

() 2009 Elsevier Masson SAS. Tous droits réservés.

Mots clés : Catastrophe ; Alerte ; Vigilance ; Routine organisationnelle ; Risque ; Expertise ; Cognition ; Apparences normales ; France

\begin{abstract}
There is a recurrent paradox: why do catastrophes always seem to be "anticipated" ex post but never ex ante? This study of the warnings issued prior to the last major natural catastrophe experienced by France- the storm of 27 December 1999 - focuses on the organizational factors accounting for the surprise occasioned by this event. Even though the French weather bureau had forecasted the storm, why did civil defense workers declare that the scale of the event came as a surprise to them? A multilevel qualitative analysis of the operation of the interorganizational weather-warning system shows that a combination of structural, contextual and individual factors turned the warnings into routine messages. The 1999 storm serves to describe an ideal
\end{abstract}

Adresse e-mail : f.dedieu@cso.cnrs.fr. 
type of a particular risk, namely the treacherous risk, which is clearly announced but disregarded because it seems so familiar.

(C) 2009 Elsevier Masson SAS. All rights reserved.

Keywords: Natural catastrophes; Storms; Warning systems; Organizational routines; Risk; Expertise; Cognition; Normalcy; France

L'usage classique de la définition d'une catastrophe est de la qualifier au regard de l'ampleur des dégâts qu'elle entraîne ${ }^{1}$. Mais si l'on se réfère à sa définition générique, « un malheur effroyable et brusque ${ }^{-2}$, on relève qu'elle possède une autre caractéristique : sa soudaineté. L'effet de surprise est, en effet, un trait commun des situations de catastrophes : si les attentats terroristes de New York du 11 septembre 2001, la canicule en France de l'été 2003 ou encore le Tsunami de décembre 2005, sont des catastrophes singulières de par leur nature, elles semblent toutes surgir par surprise.

Pour expliquer ce phénomène, la littérature sur les risques et les accidents cherche à comprendre les causes de l'échec de la prévention des catastrophes sur le long terme ou sur le court terme. Même si ces travaux divergent dans leurs approches et leurs méthodes, nombreux sont ceux qui s'accordent pour souligner les déficiences des systèmes d'alerte et de prévention. Plus précisément, un grand nombre d'auteurs se rejoignent sur le fait que les signaux précurseurs des catastrophes existaient mais ont été systématiquement mal interprétés. On a alors recours à la notion de signal faible. Initialement développée par l'épidémiologie, cette notion désigne des signaux annonciateurs d'une catastrophe qui, bien que visibles, sont généralement ignorés parce qu'ils sont très peu courants (Vaughan, 2001). Une diversité de causes permet d'expliquer pourquoi les signaux sont catégorisés comme faibles : des facteurs organisationnels et environnementaux plus larges dans les cas de l'accident de la navette Challenger (Vaughan, 1996) ${ }^{3}$ et des attentats du 11 septembre 2001 (Francart et Dufour, 2002) et des facteurs cognitifs (Lagadec et Laroche, 2005) et sectoriels (Millet, 2005) dans le cas de la canicule. Quelles que soient leurs explications, ces travaux plaident, plus ou moins explicitement, pour une thèse dite de la déficience des systèmes de prévention et d'alerte en prétendant qu' «on aurait pu savoir». Dans un récent article, André Orléan, résumait cette position en soulevant le paradoxe récurrent des situations de catastrophe : comment expliquer qu'une catastrophe semble toujours prévue ex post et jamais évitée ex ante ? (Orléan, 2008).

Nous voudrions ici contribuer à l'explication de ce paradoxe à travers l'étude de l'alerte annonçant une des deux grandes tempêtes qui toucha la France et l'Europe de l'Ouest ${ }^{4}$ en 1999, la tempête du 27 décembre 1999. Dernières grandes catastrophes naturelles que la France ait

\footnotetext{
${ }^{1}$ C'est le cas de la définition proposée par le comité français pour la Décennie internationale pour la réduction des catastrophes (DIPCN), à savoir : « une rupture grave du fonctionnement d'une communauté, accompagnée d'importantes pertes humaines, matérielles ou environnementales qui dépassent sa capacité à faire face par ses propres moyens aux actions nécessaires des secours, de récupération et de construction ».

${ }^{2}$ Définition proposé par le dictionnaire Trésor de la langue française informatisée (TLFI) consultable au lien suivant : http://atilf.atilf.fr/tlf.htm.

${ }^{3}$ Dans le cas de l'accident de Challenger, ce sont les températures anormalement basses qui ont endommagé les joints et c'est le froid qui est assimilé à un signal faible. En 1985, un an avant l'accident de Challenger, les joints avaient été également endommagés par des températures à -6 et $-7^{\circ} \mathrm{C}$. Cependant, le personnel de l'agence n'avait aucune preuve que la température était effectivement en cause mais surtout, il estimait qu'il y avait peu de chance que ce record de froid se reproduise.

${ }^{4}$ La Grande-Bretagne, l'Allemagne, la Belgique, la Suisse, l'Italie et l'Espagne.
} 
connues, les deux tempêtes exceptionnelles des 26 et 27 décembre 1999, surnommées respectivement Lothar et Martin, occasionnèrent 92 victimes et entraînèrent des dégâts considérables en France avec 3,5 millions de foyers privés d'électricité pendant plusieurs semaines (Sanson et al., 2000).

Le cas de cette alerte apparaît pertinent pour prolonger la réflexion sur le paradoxe de la prévention des catastrophes, du fait de ses fortes ambiguïtés. D'un côté, si l'on se réfère au rapport ministériel Sansom, chargé de l'évaluation des secours au cours de cet épisode, on peut plaider pour la thèse de la déficience. En dénonçant les insuffisances de Météo-France à prévoir la tempête ${ }^{5}$, le rapport prônait une refonte complète du système d'alerte et de vigilance météorologique qui eut lieu en 2001 avec le système des cartes de vigilance de couleur. Mais d'un autre côté, lorsque l'on regarde les choses de plus près, l'histoire de cette alerte semble curieuse. En effet, et contrairement à la tempête de la veille, celle du 26 décembre, Météo-France a bien annoncé une tempête exceptionnelle dès la matinée du 27 décembre, soit plus de 12 heures avant qu'elle ne provoque ses premiers dégâts sur le territoire français. L'annonce de rafales à plus de $150 \mathrm{~km} / \mathrm{h}$ annoncées par les bulletins de vigilance est particulièrement alarmante pour la France, puisque ce seuil correspond à un cyclone tropical de niveau 2 selon l'échelle de Saffir-Simpson ${ }^{6}$. De plus, toujours dans la matinée du 27 décembre, les météorologues ont averti oralement leurs interlocuteurs de la sécurité civile du caractère dangereux de cette tempête. On peut donc considérer que le signal d'alerte ne peut être catalogué comme faible puisqu'il a été clairement explicité. Malgré cela, ces différentes tentatives d'alerte n'ont pas perturbé les activités routinières des services de sécurité civile qui se déclarèrent profondément surpris de l'ampleur de la catastrophe : «je pense que rien ne laissait présager une telle catastrophe » déclare notamment un préfet d'un département côtier du Sud-Ouest français particulièrement touché par la tempête.

L'étude de l'alerte précédant la tempête Martin doit nous permettre de montrer que la thèse de la déficience et des signaux faibles n'épuise pas tous les cas de figure expliquant l'effet de surprise provoqué par les catastrophes. En introduisant le concept de risque scélérat ${ }^{7}$, nous soutiendrons que si l'alerte a été ignorée, c'est en raison de la familiarité apparente du phénomène. Autrement dit, ce concept nous permettra de montrer que cette alerte a échoué parce qu'elle a été interprétée comme l'annonce d'un danger familier auquel les acteurs impliqués dans la sécurité civile sont accoutumés. Ce danger est donc «scélérat » au sens premier du terme, parce qu'il a une propension à revêtir une apparence normale. Nous avons employé ce terme notamment en référence aux travaux d'Erwing Goffman qui décrit comment les entreprises scélérates usent des apparences normales afin, précisément, de «ne pas donner l'alarme» (Goffman, 1973, p. 245). E. Goffman prend notamment l'exemple d'une famille de vacanciers qui se révèle être des trafiquants qui cherchent à passer en fraude de la drogue sous le nez des douaniers.

Pour en faire la démonstration, nous décrirons l'histoire de cette alerte ${ }^{8}$ en nous centrant, au niveau organisationnel, sur le fonctionnement de la chaîne d'alerte météorologique unissant

\footnotetext{
5 «La météo n'a pas pu prévoir de façon convenable ces deux tempêtes et en cerner toutes les conséquences » (Sanson et al., 2000, p. 18).

${ }^{6}$ L'échelle de Saffir-Simpson permet de classifier l'intensité des cyclones tropicaux se formant dans l'hémisphère ouest. Elle comprend les cinq niveaux d'intensité suivants : niveau 1, de 118 à $153 \mathrm{~km} / \mathrm{h}$; niveau 2, de 154 à $177 \mathrm{~km} / \mathrm{h}$; niveau 3 , de 178 à $210 \mathrm{~km} / \mathrm{h}$; niveau 4, de 210 à $249 \mathrm{~km} / \mathrm{h}$; niveau 5 , des vents de plus de $249 \mathrm{~km} / \mathrm{h}$. On voit, d'après cette classification, que les valeurs annoncées par les bulletins d'alertes rendent la tempête Martin proche d'un ouragan de catégorie 2 .

7 Nous remercions ici Christine Musselin pour nous avoir aiguillé sur ce terme.

8 Cet article s'appuie sur notre travail de doctorat soutenu à l'Institut d'études politiques de Paris. Ce doctorat a bénéficié du soutien financier de la fondation Maif. L'enquête empirique s'appuie sur plus de 150 entretiens semi-directifs auprès
} 
Météo-France et la sécurité civile. Nous décrirons l'histoire de cette alerte en nous plaçant tour à tour, du côté des lanceurs d'alerte, Météo-France, et de celui des différents acteurs impliqués dans la diffusion des bulletins d'alerte pour comprendre l'interprétation qu'ils en ont fait. Il s'agit des acteurs classiques de la sécurité civile (centre opérationnel de zone, préfecture, sous-préfecture, centres principaux de secours, essentiellement les sapeurs pompiers) mais aussi d'acteurs plus divers comme l'activité maritime (marins-pêcheurs, ports de loisir, etc.), les chantiers de construction, les mairies, etc.

Nous avons opté pour une démarche d'analyse de type microsociologique afin de nous placer au plus près des pratiques des acteurs. Pour cela, nous avons situé notre analyse dans un département côtier du Sud-Ouest français particulièrement touché par la tempête. Mais surtout, cette perspective méthodologique permet de développer une analyse qualitative multiniveaux inspirée des travaux de Diane Vaughan (1996). Cette dernière se propose de rendre compte de la production et de la diffusion de l'alerte à trois niveaux du dispositif interorganisationnel d'alerte météorologique : un niveau structurel, contextuel et individuel. Cette méthode offre l'avantage de restituer $e x$ ante les logiques d'action des acteurs et de saisir la dimension dynamique des dispositifs étudiés. Mais surtout, cette démarche nous permettra de comprendre comment la familiarité apparente de ce risque se construit. Nous montrerons, en effet, que la propension de ce danger à revêtir une apparence normale s'explique par la combinaison dynamique de trois facteurs qui correspondent à chacun des niveaux du dispositif interorganisationnel d'alerte météorologique étudiés. Le risque devient donc scélérat du fait de trois types de raisons : tout d'abord, nous montrerons qu'il n'existe pas ou peu de procédures adaptées à ce type de phénomène exceptionnel (raisons structurelles). Ensuite, nous verrons comment le contexte particulier de ce mois de décembre 1999 marqué notamment par le passage de la première tempête Lothar a dénaturé le caractère exceptionnel de la menace (raisons contextuelles). Enfin, nous montrerons pourquoi les rares acteurs qui ont anticipé la catastrophe n'ont pas été entendus (raisons interactionnistes).

\section{Les raisons structurelles : les alertes météorologiques sont-elles des alertes?}

Si malgré des seuils exceptionnels, l'alerte du 27 décembre donne lieu à des réactions routinières, c'est d'abord en raison de trois types de facteurs structurels et procéduraux qui concernent Météo-France et la sécurité civile : premièrement, la confusion opérée entre alerte et vigilance dans l'énoncé et dans les dispositions de la procédure d'alerte météorologique est un handicap dans l'action préventive face aux risques naturels. Deuxièmement, les modèles de calcul de Météo-France sont limités pour prévoir les phénomènes exceptionnels comme les deux tempêtes de 1999. De ce fait, si l'alerte est donnée dans un moyen terme (12 heures avant son passage), les prévisionnistes ont des doutes sur l'ampleur du phénomène et sur sa trajectoire finale. Ce n'est qu'à court terme (6 heures avant son passage) que les météorologues prennent conscience de la nature exceptionnelle de la menace. Troisièmement, et en nous plaçant du côté des acteurs de la sécurité civile, nous verrons, d'abord, que la courte échéance à laquelle a été donnée cette alerte, a directement contribué à ce qu'elle passe inaperçue parmi le vaste réseau d'acteurs recevant les avis de vigilance de Météo-France. Ensuite, si les acteurs de la sécurité civile ont perpétué leurs routines organisationnelles face à cette annonce, c'est non seulement

de Météo-France et des multiples autres acteurs impliqués dans la réception de l'alerte météorologique (ponts, marins, sécurité civile, préfectures, sous-préfectures, mairies, etc.) ainsi que l'exploitation d'une documentation variée (main courantes préfectorale, rapports d'activité, revue de presse locale ou encore les journaux intimes des acteurs). 
parce qu'ils ne disposent pas réellement de mesures adaptées à ce type de phénomène exceptionnel mais qu'en plus, leurs réactions préventives sont limitées par un principe d'économie de coûts organisationnels.

\subsection{La lutte définitionnelle entre alerte et vigilance}

Jusqu'en 1999, la procédure dite d'alerte et de vigilance météorologique était définie par la circulaire interministérielle du 2 septembre 1993. Le principe est simple: lorsqu'un phénomène climatique potentiellement dangereux est identifié, Météo-France émet des avis de vigilance et d'alerte ${ }^{9}$ selon des seuils gradués. Sur ces bases, les services de la sécurité civile régionaux $\left(\mathrm{COZ}^{10}\right)$ et départementaux (préfectures, sous-préfecture, centres principaux de secours, essentiellement les sapeurs pompiers) appliquent alors des mesures préventives adaptées aux risques annoncés. La diffusion des bulletins de vigilance ne se limite pas aux acteurs de la sécurité civile mais à un réseau d'acteur plus vaste pour lesquels la météorologie peut avoir une influence préjudiciable sur leur activité : c'est le cas de l'activité maritime (CROSS ${ }^{11}$, marins pêcheurs etc.), des chantiers de construction ou encore d'EDF et de la SNCF dans le cas étudié.

Même si le principe apparaît simple, la mise en œuvre de la procédure est plus problématique en raison des attentes et des conceptions divergentes de l'alerte qu'ont les acteurs des deux côtés de la chaîne d'alerte. Pour sa part, Météo-France considère que la diffusion de ces alertes a un rôle essentiellement informatif. Son rôle est de donner seulement une tendance de la météo, susceptible d'évoluer, compte tenu des incertitudes inhérentes à la prévision météorologique. De leur côté, la sécurité civile, comme les différents abonnés de Météo-France, attendent de MétéoFrance un message fiable qui puisse servir de support opérationnel. Cette asymétrie des attentes s'exprime dans une lutte définitionnelle autour de l'alerte qui est inscrit dans l'intitulé même de cette procédure dite d' «alerte et de vigilance». En effet, si l'on suit la distinction opérée par Francis Chateauraynaud et Didier Torny, un régime de vigilance induit « l'attention» alors que l'alerte renvoie à la décision et à l'action (Chateauraynaud et Torny, 1999). Ce serait donc le déclenchement de mesures opérationnelles concrètes qui permettrait de distinguer l'alerte de la vigilance. Or, c'est précisément sur ce point — la mise en œuvre des procédures préventives que la confusion entre alerte et vigilance s'opère puisque l'émission d'un bulletin d'alerte de Météo-France n'assujettit pas les différents destinataires à des réactions particulières. Libres à eux de réagir selon les modalités qu'ils jugent adaptées à la situation et à l'évènement annoncé. Par conséquent, le lanceur d'alerte, Météo-France, émet une alarme mais ses «récepteurs» ne savent pas systématiquement comment réagir.

\footnotetext{
${ }^{9}$ Jusqu'en 2001, la diffusion d'une alerte météorologique correspond au découpage administratif de Météo-France. Cette dernière diffusait deux types de bulletins : au niveau national, le SCEM (service central d'exploitation météorologique) diffuse le bulletin d'alerte national ALARM (alerte aux risques météorologiques exceptionnels). Ce bulletin est destiné notamment à l'échelon national de la sécurité civile, le CODISC (centre opérationnel de la direction de la sécurité civile), un organisme relevant de l'autorité du ministère de l'Intérieur. Il est émis à chaque fois qu'un événement météorologique dangereux et exceptionnel est susceptible de toucher l'ensemble du territoire national. Au niveau régional, les interlocuteurs des CMIR (centres météorologiques interrégionaux) sont les CIRCOSC (centres interrégionaux de coordination de la sécurité civile), subdivisions régionales du CODISC. Le bulletin d'alerte diffusé au niveau régional est le BRAM (bulletin régional d'alerte météorologique). Il est émis par les CMIR à destination des COZ (centres opérationnels de zone) et contrairement aux bulletins ALARM, il n'a qu'une extension régionale.

${ }^{10}$ Les centres opérationnels de zone (COZ) sont chargés de coordonner la sécurité civile à l'échelle de la zone de défense.

${ }^{11}$ Les centres régionaux opérationnels de surveillance et de sauvetage (CROSS) dépendent du ministère de l'Équipement et des Transports. Ils sont dirigés par des administrateurs des Affaires maritimes (représentants permanents du préfet maritime) et sont constitués de personnels militaires de la Marine nationale et des Affaires maritimes.
} 
De plus, le système d'alerte météorologique confond vigilance et alerte non seulement dans les termes mais surtout dans les contraintes qu'il se donne : a priori il semble s'apparenter à un système de vigilance puisque l'émission des bulletins n'a pas de portée opérationnelle. Pourtant, du point de vue de Météo-France, le système prévoit deux dispositions qui évoquent un schéma d'alerte. Tout d'abord, la procédure comprend des niveaux d'alerte permettant de traduire une graduation des dangers annoncés ${ }^{12}$. Ensuite, la diffusion des bulletins d'alerte fait l'objet, pour les météorologues, d'une évaluation en interne régulière puisque la sécurité civile est une des missions institutionnelles de Météo-France. Tous les trois mois, les alertes diffusées par MétéoFrance font l'objet d'une évaluation conjointe par un comité interministériel national constitué du service national de Météo-France et des officiers de la sécurité civile. Dans cette évaluation, les deux acteurs prennent en compte la part d'incertitude inhérente à la prévision. La sécurité civile et Météo-France instaurent une règle informelle qui prévoie une marge de tolérance d'environ $15 \%$ sur la diffusion de «fausses » alertes (Corvalan, 2005). Mais l'instauration de ce seuil n'exonère pas les débats dans lesquels les deux services s'opposent sur ce qu'est une alerte avérée ou non : Météo-France considère que la diffusion d'une alerte est justifiée lorsque la mesure réelle d'un phénomène ${ }^{13}$ est conforme aux seuils annoncés dans leurs prévisions. De son côté, la sécurité civile apprécie l'efficacité d'une alerte à la hauteur des dégâts qu'a engendré le phénomène annoncé ${ }^{14}$.

Précisons ici un point important pour la suite de notre propos. En raison du statut d'organisme public, les services de Météo-France sont assujettis à une «obligation de moyen » et non pas à une obligation de résultats. Ce principe juridique inscrit dans les statuts de Météo-France prévoit que «tous les moyens dont disposent les organismes de Météo-France doivent être mis en œuvre» dans la production de la prévision. Autrement dit, Météo-France n'est pas soumis à une obligation de résultat qui la contraindrait à fournir une prévision exacte à $100 \%$. Ses services doivent faire la démonstration qu'ils utilisent, au maximum de leur diligence et de leur expertise, l'ensemble des moyens dont ils disposent. La responsabilité civile ou pénale de Météo-France peut être engagée, s'il est établi que l'organisme n'a pas su mobiliser l'ensemble des moyens dont il disposait.

Aussi, même si l'obligation de moyens semble exonérer Météo-France de toute exigence de résultat, les évaluations communes avec la sécurité civile constituent une contrainte réelle pour Météo-France et plus particulièrement pour l'échelon régional des CMIR puisqu'ils sont les principaux diffuseurs d'alerte météorologiques. Malgré les interprétations divergentes de l'alerte, le seuil informel de $15 \%$ de «fausses alertes » est surtout apprécié en fonction de la définition qu'en donne la sécurité civile, c'est-à-dire une alerte qui précède des dégâts notables. Ainsi, lorsqu'ils diffusent une série trop importante de «fausses alertes » selon cette définition, les prévisionnistes doivent se justifier auprès des acteurs de la sécurité civile mais surtout auprès de la plus haute hiérarchie du SCEM, le directeur de la prévision. Aussi, les prévisionnistes considèrent être astreints à une forme d'obligation de résultat dans la diffusion des avis de vigilance ${ }^{15}$.

\footnotetext{
12 Les bulletins BRAM ou ALERTE face aux vents étaient déclenchés à partir du seuil de $100 \mathrm{~km} / \mathrm{h}$ dans les terres et $120 \mathrm{~km} / \mathrm{h}$ sur les côtes. La nouvelle procédure de 2001 dite des «cartes de vigilance » a conservé ces différents seuils d'alerte, prévoyant ainsi les niveaux vert, jaune, orange et rouge.

13 Cela peut être la hauteur de neige, la puissance des vents ou encore le degré de pluviométrie par exemple.

14 Par exemple, pour eux, une alerte météorologique est jugée pertinente lorsqu'un bulletin d'alerte annonce des orages violents entrainant la destruction de plusieurs campings, comme ce fut le cas dans les Landes au cours de l'été 2001.

15 Depuis les tempêtes de 1999, Météo-France et la sécurité civile ont définitivement entériné cet aspect. Trois objectifs, qui se rapprochent d'une obligation de résultat, sont désormais formellement inscrits dans la nouvelle procédure d'alerte
} 
La chaîne d'alerte météorologique se présente donc comme un système de vigilance ayant les contraintes d'un système d'alerte. Alors que tout repose sur l'étroitesse de la relation entre MétéoFrance et la sécurité civile au moment des alertes, la procédure ne prévoit pas de dispositions qui instaureraient une quelconque interdépendance opérationnelle et juridique entre les deux acteurs. Au contraire, il existe un «vide » réglementaire qui fait que les responsabilités sont clairement séparées entre eux. Ce «flou»d'appréciation entre alerte et vigilance permet d'ailleurs à la sécurité civile de se défausser de la responsabilité de non-anticipation de catastrophes de grande ampleur. C'est dans cet espace «non réglementé » et dépourvu de contraintes opérationnelles entre la diffusion et la réception de l'alerte que s'exerce la vigilance face à la future catastrophe.

«Notre travail c'est avant tout d'informer et de le faire sur des bases les plus précises possible. On ne peut pas se substituer aux services pour leur dire ce qu'ils doivent faire, c'est pour cela que l'on travaille étroitement avec la sécurité civile. Alors est-ce qu'il s'agit d'une alerte ? Tout dépend de ce que vous entendez par-là, si c'est de dire "attention quelque chose se prépare et ça peut être dangereux" alors il s'agit d'une alerte. Mais si c'est dire "vous allez avoir tel phénomène qui va vous tomber dessus ici précisément", alors non il ne s'agit pas d'une alerte. La prévision météo ne peut pas toujours se faire comme ça. On peut donner l'alerte sans que cela implique forcément des interventions sur le terrain ou qu'il y ait des blessés » Chef prévisionniste CMIR.

\subsection{Les limites techniques des modèles numériques de calcul de Météo-France}

Même si elle est diffusée tôt dans la matinée du 27 décembre, l'alerte annonçant la tempête est remplie d'incertitudes en raison des limites techniques des modèles numériques de Météo-France. De ce fait, le caractère exceptionnel du phénomène n'est appréhendé que dans un court terme, six heures avant ses premiers dégâts en France.

La prévision météorologique est un processus complexe qui mêle étroitement la simulation du temps par les modèles de calcul et les observations de l'atmosphère par les météorologues. Mais, du fait de leur programmation, les modèles, et notamment le modèle de référence de Météo-France ARPEGE, ne peuvent pas restituer les phénomènes climatiques qui se forment très rapidement et ceux qui présentent des anomalies de formation très prononcées au regard des standards normalement observés. Les deux tempêtes de 1999, Lothar et Martin correspondent à ces deux critères. Elles se sont constituées très rapidement et de surcroît, à des endroits inhabituels au regard des standards de formation des tempêtes d'hiver classiques. Mais les anomalies de formation des deux tempêtes de 1999 sont à chaque fois spécifiques. La tempête du 26 décembre, Lothar, s'est formée en moins de 12 heures, une vitesse exceptionnelle en comparaison des 48 à 72 heures généralement nécessaires. La tempête Martin, elle, a eu un stade de formation plus classique. Elle s'est développée en 48 heures, un rythme plus proche de celui des tempêtes classiques. L'anomalie de cette tempête réside plutôt dans sa trajectoire, continuellement mouvante tout au long de sa phase de formation.

Du fait de ces anomalies de formation, les tempêtes constituent une «cause accidentelle» (Desrosières, 2000) dans la programmation des modèles de calcul : calculée à partir de moyennes,

et de vigilance. Il s'agit : 1 , ne diffuser aucune fausse alerte pour ne pas décrédibiliser les bulletins; 2 , ne pas produire de non-détection; 3, donner le bulletin avec une anticipation de trois heures minimum. 
la prévision de ces phénomènes anormaux enregistre des écarts importants ${ }^{16}$ entre la progression de leurs valeurs et les ébauches initiales. En se creusant en si peu de temps, Lothar a circulé à une vitesse inhabituelle sur l'Atlantique, ce qui a totalement pris de court les météorologues. Elle n'est apparue qu'au tout dernier moment, dans les observations de temps réel, deux heures avant qu'elle n'apparaisse sur la France, si bien que l'alerte a été donnée au moment où elle commençait à s'abattre sur la France. Pour la tempête Martin, le problème est tout autre. Elle est identifiée par les modèles dans la nuit du 26 au 27 décembre. Les résultats des différents modèles convergent sur le scénario d'une forte cyclogenèse sur la France pour la fin de journée du 27 décembre. Les modèles restituent une tempête dont les rafales atteindront les $150 \mathrm{~km} / \mathrm{h}$. Mais au moment du lancement des premières alertes de tempête dans la matinée du 27 décembre, les bulletins diffusés au niveau national (bulletin ALARM 7.1 diffusé à $8 \mathrm{~h} 50$ ) et les bulletins diffusés au niveau régional (bulletins BRAM 37.1 diffusé à 9 heures) ${ }^{17}$, les prévisionnistes ne peuvent pas se prononcer exactement sur l'ampleur de la tempête ${ }^{18}$ et sur la trajectoire exacte qu'elle va suivre. En effet et toujours en raison des estimations «moyennes» fournies par les modèles, la prévision du rayon géographique qui va subir l'impact de l'événement est trop large pour pouvoir déterminer, à moyen terme, quels seront les territoires les plus concernés. Du fait de ces limites techniques, la qualification de la menace demeure incertaine dans un moyen terme, c'est-à-dire 12 heures avant que la tempête ne touche la France. C'est grâce aux observations de l'après-midi, faites entre 14 et 16 heures, que les météorologues parviennent à mesurer plus précisément l'ampleur du creusement de la tempête (et donc la force des vents) et sa trajectoire finale. Les rafales de vent dépasseront les seuils de $150 \mathrm{~km} / \mathrm{h}$ qui ont été initialement annoncés et la tempête touchera le Sud de la France.

Du fait de l'incertitude technique qui entoure la prévision météorologique de la tempête, la menace n'est dévoilée qu'à très court terme. Mais avant tout, c'est la compatibilité des caractéristiques du phénomène avec le système technique de prévision météorologique qui détermine le délai qui est laissé à la prévention. Si contrairement à Lothar, la tempête Martin a pu être prévue, c'est parce que ses anomalies de formation étaient moins prononcées que celle de la veille.

\subsection{Vigilance et routines organisationnelles}

Plaçons nous à présent du côté des récepteurs de l'alerte. Le 27 décembre 1999, les avis de vigilance exceptionnels donnent lieu à l'application de mesures routinières parmi le vaste réseau d'acteurs inclus dans le cercle de diffusion de Météo-France. Mais la routine n'équivaut pas systématiquement à la passivité. Si certains comme les autorités départementales (préfet, maires principalement), EDF ou la SNCF n'ont pas réagi, d'autres acteurs comme l'activité maritime (les ports de tourisme et les marins-pêcheurs), les aéroports ou les chantiers de construction ont appliqué des mesures préventives routinières à réception des bulletins. Si les avis de vigilance provoquent uniquement des réactions routinières c'est en raison d'un principe d'économie de coûts organisationnels exacerbé par le délai court auquel est donné cette alerte. Développons ces

\footnotetext{
16 Ce que nous appelons l'écart entre les données peut concerner, par exemple, la position d'altitude de la dépression qui évolue, entre les deux ébauches, dans un sens trop éloigné des paramétrages.

17 Dans la journée du 27 décembre, les BRAM concernent les régions du Poitou-Charentes, d'Aquitaine, de MidiPyrénées, du Limousin, d'Auvergne, Rhône-Alpes, de Languedoc-Roussillon, de Provence-Alpes-Côte d'Azur et de la Corse.

${ }^{18}$ La force des vents est mesurée par son creusement dépressionnaire.
} 
deux points qui permettent de comprendre pourquoi les routines ont été perpétuées au cours de cet épisode.

Premier point, prise dans son rapport à la vigilance, la routine organisationnelle s'explique par une économie implicite de coûts organisationnels qui régit le fonctionnement quotidien des organisations (Ménard, 1997). Il s'agit, plus précisément, d'un rapport entre les coûts « attendus » face aux vents et les coûts de «mise en œuvre» des procédures préventives. Les coûts «attendus » désignent les conséquences ou les perturbations prévisibles qu'occasionneraient les vents violents pour l'activité de l'organisation. Ils sont de nature diverse : ils peuvent être juridiques, économiques ou encore liés à la sécurité des agents ou des usagers de l'organisation. Les coûts de mise en œuvre, quant à eux, sont ceux qu'occasionne l'application concrète des mesures préventives. Ce rapport est plus ou moins important selon les cas, ce qui explique pourquoi certains ont réagi à la lecture des bulletins de vigilance et d'autres pas. Pour illustrer ces variations, prenons deux cas opposés.

Tout d'abord, prenons le cas des exploitants pêcheurs du département qui ont appliqué des mesures préventives dans la matinée du 27 décembre. Avant d'entamer une sortie en mer, les pêcheurs se tiennent toujours informés des conditions climatiques sur le grand large grâce à trois bulletins journaliers transmis par le CROSS. Le 27 décembre, des bulletins d'alerte spéciaux, annonçant des vents de force 9 à 10 sur mer, sont diffusés à intervalles réguliers à partir de $9 \mathrm{~h} 30$ environ. Ces valeurs exceptionnelles dissuadent les marins-pêcheurs ${ }^{19}$ de prendre la mer ce jour-là. En effet, les coûts « attendus » sont particulièrement élevés. Ces coûts sont d'abord liés à leur propre sécurité, puisque naviguer au milieu d'une tempête correspondant au seuil maximal d'avis de tempête sur mer (des vents de force 9), s'avérerait particulièrement dangereux. Mais ces coûts sont aussi économiques. Ils sont, plus précisément, liés aux régimes d'assurance ${ }^{20} \mathrm{de}$ ces professionnels. Effectivement, si les marins-pêcheurs s'aventuraient à prendre la mer malgré des avis de vigilance alarmants, les assurances ne couvriraient pas les frais occasionnés par des conduites aussi risquées. D'un autre côté, les coûts de mise en œuvre de ces mesures préventives ne se traduisent que par une journée d'exploitation perdue. Dans leur cas, le déséquilibre entre ces deux types de coûts est tellement important qu'ils n'hésitent pas à rester à quai. Ces journées sont consacrées généralement à des tâches de manutention diverses ou à la mise à jour de la comptabilité.

Inversement, d'autres acteurs comme la SNCF ou les élus communaux qui seront pourtant particulièrement touchés par la tempête ne réagissent pas au BRAM soit parce qu'ils ne disposent pas de procédures adaptées, soit parce qu'il s'avérerait trop coûteux d'appliquer des mesures préventives. Tout d'abord, réagir face à ce type de phénomène, implique que les acteurs disposent de solutions opérationnelles adaptées qui permettent de faire face à des vents de cette ampleur. C'est le cas des services d'exploitation de la SNCF chargés de l'entretien des voies et qui se trouveront directement impliqués dans les secours puisque des trains seront bloqués en pleine voie la nuit de la tempête. Pourtant, dans la matinée du 27 décembre, les agents SNCF des centres d'exploitation ne prêtent pas une attention particulière aux différents BRAM diffusés au cours de la journée. S'ils n'y prêtent pas vraiment attention, c'est que l'annonce de vents violents

\footnotetext{
${ }^{19}$ Nous basons ce constat sur les entretiens que nous avons réalisés avec les marins-pêcheurs rattachés au principal port de pêche du département. Mais nous pouvons élargir ce constat au département puisque dans les différents rapports et documents établissant le bilan des dégâts de la tempête, nous n'avons pas relevé d'incidents autour de l'activité maritime hormis les dégâts matériels dans les ports.

${ }^{20}$ Ici encore, nous nous référons aux entretiens que nous avons réalisés auprès des marins-pêcheurs. Nous ne nous sommes pas livrés à l'examen précis des contrats d'assurance professionnels de ces navigants.
} 
n'est pas associée à la possibilité de perturbations sur le trafic SNCF. Autrement dit, les coûts « attendus » occasionnés par les vents violents sont faibles. Il n'existe pas de correspondance entre ce signal et une réaction préventive, parce que ce type de risque ne correspond à aucun dommage attendu et qu' aucune mesure particulière n'est prévue pour y faire face. Les autorités préfectorales et municipales, pourtant responsables ${ }^{21}$ de la sécurité de la population dans le département, se trouvent également dépourvus de solutions préventives face à ce type de phénomène exceptionnel. Ils peuvent s'appuyer sur les plans de prévention de long terme (les PPR $^{22}$ ) mais ceux-ci ne prévoient pas de dispositions particulières pour les tempêtes. De même, les plans de secours d'urgence comme le plan ORSEC ${ }^{23}$ ou le plan rouge qui sont du ressort de la préfecture servent d'appui à l'action dans l'urgence et n'ont pas, par conséquent, de vocation préventive.

Tout se passe donc comme si le fait de ne pas disposer de procédures face à ce risque concourait à détourner la vigilance de ces acteurs. On retrouverait ici les conclusions de Karl E. Weick sur le rôle du formatage cognitif joué par les mesures techniques et les procédures des organisations sur l'attention que portent les acteurs aux signaux de l'environnement (Weick, 1995) ${ }^{24}$. Mais cette explication cognitive sur laquelle nous reviendrons plus bas nous semble incomplète puisque l'exercice de la vigilance répond également à des contraintes opérationnelles. Une analyse en termes de coûts organisationnels montre que la sélection des signaux de l'environnement obéit à un besoin de stabilité de l'organisation et pas seulement à un formatage cognitif joué par le design organisationnel et technique des organisations. Le cas des services de maintenance d'EDF ${ }^{25}$ chargés de l'exploitation, de l'entretien et de la sécurité du réseau de transport électrique (RTE) est éloquent de ce point de vue. Alors que les vitesses de vents annoncées (des pointes à $150 \mathrm{~km} / \mathrm{h}$ ) laissaient imaginer de nombreuses coupures sur le «transport» aérien, les centres de maintenance ont poursuivi leurs activités routinières le 27 décembre. Ils ont reconduit leur système d'astreinte ordinaire qui prévoit une permanence continue des techniciens la nuit et les week-ends. C'est du fait de ce système qu'il n'existe pas à EDF de solutions préventives adaptées pour des tempêtes

\footnotetext{
${ }^{21}$ Comme le précise Christian Frémaux, les maires sont directement concernés par les risques qui touchent à la sécurité publique, même si ceux-ci sont exceptionnels. Selon l'auteur, la responsabilité des maires en cas de catastrophes peut être engagée sur le plan civil voire sur le plan pénal. Il précise: « dès qu'un risque existe fut-il minime, improbable voire « virtuel», le maire a le devoir d'agir, quitte à s'exposer à des recours contentieux par les intéressés » (Frémaux, 2002, p. 44). De manière similaire aux élus municipaux, la responsabilité civile et pénale du préfet peut être engagée s'il est établi que des décès occasionnés par une catastrophe industrielle et naturelle sont associés aux carences du contrôle des services de l'État ou plus précisément à l'absence de vigilance.

${ }^{22}$ Les plans de prévention des risques (PPR) ont pour objectifs d'améliorer la sécurité des personnes exposées aux risques, de limiter les dommages aux biens et aux activités soumises aux risques. Pour mettre en œuvre ces objectifs, le PPR doit : 1, délimiter les zones exposées aux risques en tenant compte de la nature et de l'intensité du risque encouru ; 2 , définir sur ces zones des mesures d'interdiction ou de prescriptions vis-à-vis des constructions, ouvrages, aménagements ou exploitations qui pourraient s'y développer.

${ }^{23}$ Le plan ORSEC est une organisation des secours créée par instruction ministérielle du 5 février 1952, refondée récemment par la loi de modernisation de la sécurité civile du 13 août 2004.

${ }^{24}$ Lorsqu'il cherche à saisir les « occasions de sensemaking», K. Weick s'interroge sur la manière dont les individus «trient» les signaux (les cues) de l'environnement. Il part du constat que les environnements se caractérisent par leur complexité, leur turbulence et par la surcharge d'informations potentielles qu'ils renferment. Ces trois caractéristiques rendent donc l'environnement profondément incertain, ce qui pousse les organisations à se doter de mesures ou de techniques qui permettent de traiter des informations extérieures, généralement ambiguës et floues. C'est donc grâce à ces techniques et à ces mesures que l'individu parvient à ne pas se noyer dans l'incertitude de l'environnement. Mais, en contrepartie, ces systèmes créent les conditions pour que l'individu remarque moins de signes, ou plutôt qu'il soit capable de donner du sens à de moins en moins de choses.

25 On trouve, dans le département étudié, les centres d'exploitation dans les principales villes du département. C'est de cette manière, qu'EDF parvient à couvrir l'ensemble des lignes aériennes du département.
} 
peu ordinaires. Il a été envisagé, notamment après le passage des tempêtes de 1999, de créer des cellules d'intervention mobiles qui seraient capables d'intervenir à tout moment partout sur le territoire pour renforcer les équipes locales. Mais entretenir une cellule permanente de techniciens s'avérerait trop coûteux au regard de la faiblesse du nombre de tempêtes aussi destructrices que celles de 1999. En d'autres termes, le rapport entre les coûts de mise en œuvre est trop déséquilibré par rapport aux coûts attendus face aux tempêtes de cette ampleur. La projection de ce rapport défavorable entre coûts attendus et coûts de mise en œuvre fait que le système de prévention à EDF n'a pas évolué. Par conséquent, comme en 1999, si une tempête de cette ampleur touchait la France aujourd' hui, les agents EDF n' auraient pas d' autre choix que d'attendre que les destructions interviennent pour agir.

Deuxième point, la courte échéance à laquelle est donnée l'alerte contribue directement à renforcer les routines organisationnelles. Nous l'avons vu, ce n'est qu'en milieu d'après-midi que les météorologues disposent de données plus précises sur la puissance des vents et surtout sur la trajectoire de Martin. De plus et du fait de cette courte échéance, les météorologues ne disposent pas de suffisamment de temps pour prévenir l'ensemble des acteurs de la sécurité civile. Ce sont les bulletins d'alerte régionaux et nationaux de l'après midi, le BRAM 37.5 diffusé à $16 \mathrm{~h} 10$ et le bulletin ALARM 7.3 diffusé à 17 heures $^{26}$ qui permirent de préciser l'imminence du danger. Lorsque ces bulletins sont émis, on est à moins de deux heures de l'apparition de la tempête dans le département. Or, la circulation de ces bulletins dans le circuit administratif centralisé prend du temps, ce qui retarde leur réception par les autorités départementales et en premier lieu les mairies: le BRAM 37.5 est rédigé au centre régional à $15 \mathrm{~h} 10^{27}$. Une fois la procédure d'autorisation interne à Météo-France effectuée ${ }^{28}$, il est émis à $16 \mathrm{~h} 10^{29}$. Le service de sécurité civile de la préfecture (SIACPC) le reçoit 20 minutes plus tard à $16 \mathrm{~h} 30$. Vers 17 heures ${ }^{30}$, ce bulletin s'accompagne d'un avertissement oral, du directeur départemental de Météo-France qui avertit le service du caractère dangereux du phénomène. Conformément à la procédure, le SCIACPC débute à son tour la diffusion du bulletin à partir de $17 \mathrm{~h} 20^{31}$. Les fax sont émis selon un ordre de diffusion particulier. Ce sont les petites communes qui reçoivent ces bulletins en dernier via les sous-préfectures. Certaines petites mairies côtières qui seront particulièrement touchées par la tempête reçoivent le fax d'alerte à $17 \mathrm{~h} 45^{32}$ de la sous-préfecture de Rochefort. À cette heure, non seulement la tempête a déjà débuté mais en plus les mairies sont fermées. En résumé, il s'est écoulé plus de deux heures et demie entre la rédaction du bulletin (15 h 10) à Météo-France et sa réception dans les communes $(17 \mathrm{~h} 45)$.

Pour la sécurité civile, une alerte donnée à une échéance aussi brève restreint un peu plus les possibilités d'action préventive et, consécutivement, renforce les réactions routinières. En effet, sans solutions opérationnelles réellement adaptées, la réactivité face à ces phénomènes peu ordinaires repose sur la capacité d'innovation des acteurs. Or, dans un délai aussi court, celles-ci auraient été compliquées à mettre en œuvre. Si l'on s'essaie à définir ce qui «aurait pu être fait», on peut retenir, outre les mesures de confinement actuellement préconisées ${ }^{33}$, le

\footnotetext{
26 Source BRAM et ALARM : main courante préfectorale ORSEC.

27 Ibid.

${ }^{28}$ Il s'agit d'une autorisation formelle signée par le chef prévisionniste du centre régional et du SCEM.

29 Source : main courante préfectorale ORSEC.

${ }^{30}$ L'heure nous a été donnée par le membre du SIDPC qui a reçu le coup de téléphone.

31 Source : main courante préfectorale ORSEC.

32 Ibid.

33 Il s'agit désormais des mesures préconisées par Météo-France lors des tempêtes de forte ampleur. La vigilance orange face aux vents violents prévoit les recommandations suivantes pour la population : «Limitez vos déplacements. Limitez
} 
scénario de l'évacuation des communes du littoral ayant subi le raz-de-marée provoqué par la tempête. Mais on imagine que l'organisation improvisée de l'évacuation de la population des communes côtières en deux heures aurait été particulièrement problématique. Autrement dit, à supposer que les acteurs soient parvenus à imaginer ces mesures préventives, ils ne disposent pas de suffisamment de temps pour d'abord les créer et ensuite les appliquer.

En conséquence, il ressort de ce premier niveau d' analyse que ce qui peut apparaître a posteriori comme une absence de vigilance face à un tel risque correspond à une série de limites structurelles qui concernent les différents acteurs qui composent la chaîne d'alerte météorologique. Pour MétéoFrance, il s'agit des limites techniques de ses modèles de prévision qui permettent d'appréhender ce type de phénomène exceptionnel uniquement dans un court terme. Si les organisations recevant ces avis de vigilance ne réagissent pas de manière anticipée à l'annonce de la tempête, c'est soit parce qu'elles ne disposent pas des moyens nécessaires ou que ceux-ci seraient trop coûteux pour pouvoir le faire régulièrement. De plus, le fait que l'annonce de la catastrophe intervienne dans un court terme restreint encore un peu plus les possibilités de réactions préventives et renforce les réactions routinières. Enfin, la lutte définitionnelle autour de l'alerte entretenue conjointement par Météo-France et la sécurité civile handicape un peu plus l'action préventive puisqu'il n'existe pas de solutions opérationnelles qui permettraient de passer d'un régime de vigilance à un régime d'alerte. En somme, la prévention de ce type de phénomène est limitée dans son fondement même, puisque les mesures disponibles sont trop restreintes pour pouvoir faire face à de tels risques.

Les limites structurelles de la prévention que nous venons de pointer questionnent la définition de l'alerte et par conséquent son évaluation dans le cas des grandes catastrophes naturelles. Nous avons établi plus haut que l'alerte impliquait, de manière substantive, qu'une réaction soit rattachée à l'avertissement lancé ${ }^{34}$. Or, nous avons pu montrer que, dans le cas de la tempête, il n'existe pas, ou peu, de mesures préétablies face à un événement de cette ampleur. Dès lors, peut-on parler d'une déficience de l'alerte dans cet épisode comme l'a établi le rapport ministériel Sanson et al. (2000) ? On serait tenté de répondre par la négative puisqu'il y a bien eu vigilance puis alerte mais selon des modes «normaux». Mais l'on pourrait tout aussi bien soutenir l'inverse, puisque le phénomène n'a pas été anticipé comme tel. En somme, les résultats apportés à ce premier niveau d'analyse soulignent la forte ambiguiité qui se loge dans la définition que l'on donne à une alerte lors des grandes catastrophes naturelles et toute la difficulté qu'il y aurait à évaluer ce que serait une alerte réussie.

\section{Les raisons contextuelles : la menace diluée dans les apparences normales}

Le contexte d'action particulier de ce mois de décembre 1999 a contribué à dénaturer la menace. Les effets contextuels ont pesé à la fois sur la diffusion orale de l'alerte par Météo-France et sur son interprétation par les différents acteurs de la sécurité civile. L'échec de la prévision de la première tempête, la veille, et une répétition de cyclogenèses qui n'achèvent pas leur formation (les « faux départs » sur l'Atlantique) des jours précédents, ont conduit les prévisionnistes de Météo-France à

votre vitesse sur route et autoroute, en particulier si vous conduisez un véhicule ou attelage sensible aux effets du vent. Ne vous promenez pas en forêt [et sur le littoral]. En ville, soyez vigilants face aux chutes possibles d'objets divers. N'intervenez pas sur les toitures et ne touchez en aucun cas à des fils électriques tombés au sol. Rangez ou fixez les objets sensibles aux effets du vent ou susceptibles d'être endommagés » (souce : www.meteo.fr).

${ }^{34}$ La définition du dictionnaire Trésor de la langue française informatisée (TLFI) renvoie à ce présupposé : «Alerter qqn. L'avertir d'un danger, d'une situation critique ou, personne ext., d'un phénomène anormal, d'une difficulté quelconque, pour que soit éventuellement prises des mesures de vigilance, d'aide ou d'intervention ». 
se montrer très prudents dans la transmission de l'alerte. Ensuite, si les acteurs de la sécurité civile du territoire étudié n'ont pas réellement cru à la possibilité d'une catastrophe c'est parce qu'ils ont vu dans la tempête qui approchait un épisode semblable à ceux qu'ils subissent habituellement à cette période de l'année. Ce sentiment a, du reste, été conforté par la répétition des «fausses » alertes des jours précédents.

\subsection{Le contexte parasite la transmission orale de l'alerte}

La prévision à court terme de la tempête ne se limite pas aux limites techniques des modèles numériques. Les contraintes contextuelles et organisationnelles internes à Météo-France ont également pesé de tout leur poids au cours de cet épisode. Reprenons le fil de la description de la prévision de la tempête pour en faire la démonstration.

Dans la nuit du 26 au 27 décembre 1999, et avant les modèles numériques, la tempête est repérée grâce à l'intuition ${ }^{35} \mathrm{~d}$ 'une responsable prévisionniste du SCEM. En effet, les changements brutaux de directions que connaît cette tempête depuis la vieille, lui rappelle la trajectoire de la tempête de février $1996^{36}$ qu'elle avait elle-même pu observer : comme cette tempête, la circulation de Martin est continuellement oscillante et connaît des stades d'évolution très rapides. Ce rapprochement lui permet de croire que cette tempête pourrait brusquement changer de son cap actuel pour toucher la France et plus particulièrement le Sud de la France. Dans le choix du scénario de la prévision qui est élaboré en concertation avec les services régionaux météorologiques (les CMIR), elle préconise aux prévisionnistes du centre du Sud-Ouest d'alerter la sécurité civile du danger que représente cette tempête. Mais lors de la conférence téléphonique de 21 heures où les échelons nationaux et régionaux de Météo-France s'accordent sur le scénario de la prévision du lendemain, un désaccord oppose les prévisionnistes du SCEM à ceux du CMIR sur la tempête. Le CMIR refuse de lancer une alerte sur la seule base du jugement de la prévisionniste arguant que les modèles ne prévoient pas de tempête. Responsable de la prévision de leur territoire, le CMIR a le dernier mot sur le choix du scénario de la prévision et obtient par conséquent gain de cause dans ce litige.

Pour comprendre cette attitude de repli rigide sur les résultats des modèles dans ce cas, il faut préciser les contradictions induites par les modes d'évaluation des alertes météorologiques élaborés conjointement avec la sécurité civile : même s'ils ont, globalement, tendance à maximiser les seuils pour ne pas passer à côté de phénomènes potentiellement dangereux, les prévisionnistes se montrent, à l'inverse, souvent plus prudents après une succession rapprochée de «fausses alertes ». Plus précisément, lorsque le seuil toléré de $15 \%$ de «fausses alertes » est dépassé, le CMIR est plus réticent à les diffuser, si elles ne reposent pas sur des bases solides, c'est-à-dire s'il n'y a pas convergence entre les modèles. En cas de diffusion d'alertes trop répétées et d'incertitude trop forte dans la prévision, les prévisionnistes se conformeront aux résultats du modèle Arpege, toujours pour assurer l'obligation de moyens. En somme, l'obligation de moyens prime dans la diffusion de l'alerte: si les évaluations conjointes de l'alerte constituent bien une contrainte pour les prévisionnistes, Météo-France cherchera avant tout à satisfaire son obligation de moyens, quitte à mécontenter les services de sécurité civile (Corvalan, 2005).

\footnotetext{
35 Nous entendons par intuition le sens que lui donne Michael Polanyi, le pressentiment d'une découverte (Polanyi, 1963).

${ }^{36}$ La tempête de 1996 a touché la côte ouest de la France et plus particulièrement les Landes et la Bretagne (Finistère) (Baleste et al., 2001).
} 
C'est ce même mécanisme qui a joué dans l'épisode de la tempête Martin. En décembre 1999, les prévisionnistes du CMIR du Sud-Ouest se trouvent dans une position delicate : du fait de la diffusion répétée des précédents BRAM (trois en trois jours), les prévisionnistes avaient, en quelque sorte, épuisé leur crédit de $15 \%$ de fausse alerte. Ils craignent alors de se trouver en porte-à-faux vis-à-vis de leur hiérarchie lorsqu'ils devront justifier leur prévision lors des bilans effectués avec la sécurité civile que nous mentionnions précédemment. La répétition des fausses alertes dans les jours qui ont précédé Martin incite donc les prévisionnistes régionaux à se montrer prudents. Avant de lancer l'alerte, ils choisissent d'attendre les dernières évolutions du phénomène.

Mais surtout, leur prudence est renforcée par l'échec de la prévision de la tempête Lothar de la vieille et de la pression médiatique que cela a occasionnée sur les services de Météo-France. En effet, si les CMIR avaient choisi de diffuser plus d'alertes à un moment où l'opinion médiatique pointe du doigt leur incapacité à anticiper la première tempête, ils auraient été, selon eux, immédiatement accusés d'appliquer la «politique du parapluie». Autrement dit, s'ils émettent une nouvelle alerte à la tempête, ils craignent qu' on leur reproche de vouloir rattraper leur erreur de la veille de manière trop flagrante. À cela s'ajoute le fait que les prévisionnistes ont du mal à croire que la France puisse être touchée coup sur coup par deux tempêtes exceptionnelles : un second phénomène de cette ampleur leur semble tellement improbable que cela ne fait qu'encourager leurs réticences à lancer l'alerte. Toutefois, ces obstacles sont contrebalancés par la position hiérarchique élevée de la chef prévisionniste. Du fait de sa fonction, son avis n'est pas complètement ignoré dans la production du scénario de la prévision du 27 décembre. Même s'ils récusent sa prévision dans la conférence de 21 heures du 26 décembre, les prévisionnistes régionaux lui assurent qu'ils accorderont une attention toute particulière à l'évolution de la cyclogenèse.

Ce n'est qu'au cours de la nuit du 26 au 27, lorsque les modèles commencent à restituer le scénario d'une forte cyclogenèse sur la France, que les prévisionnistes régionaux constatent que la prédiction de la chef prévisionniste est confirmée. Dans les premières heures de la matinée, ils décident donc de diffuser les premiers bulletins d'alerte au niveau régional (bulletin BRAM 37.1 diffusé à 8 h 50) mais aussi au niveau national (bulletin ALARM 7.1 diffusé à 9 heures). Malgré l'importance du seuil de vitesse de vent annoncé dans ces avis de vigilance, la phase orale de l'alerte est à nouveau parasitée par les défaillances de la prévision de la première tempête. En effet, dans leurs avertissements à la sécurité civile, ils ne peuvent pas surestimer un phénomène qui serait mineur par rapport à Lothar mais dans le même temps, ils ne doivent pas sous-estimer le caractère potentiellement dangereux du phénomène. Les prévisionnistes résolvent ce dilemme en optant pour une prévision « prudente » du phénomène à moyen terme. Ainsi, alors que l'on pourrait croire que le fait que le territoire ait été précédemment touché par une catastrophe équivalente puisse accréditer le scénario d'une seconde catastrophe, c'est l'inverse qui se produit: la tempête Lothar a handicapé un peu plus la prévision de Martin.

«Si la première tempête a eu une influence ça aurait été plutôt de minimiser la deuxième. Pourquoi ? Parce que l'on ne pouvait pas se tromper une deuxième fois comme on l'avait fait la première. Imaginez si la deuxième on l'annonce trop forte. On nous dira ensuite "vous vous êtes trompés une première fois, c'est pour ça qu'ensuite vous prévoyez des tempêtes tout le temps". D'ailleurs, c'est pour cela que l'on a attendu le 27 pour diffuser le bulletin ALARM. On l'a diffusé sur les informations de six UTC qui montraient qu'elle était en train de se creuser. » Chef prévisionniste SCEM.

Ce n'est qu'en début d'après-midi, entre 14 et 16 heures, lorsqu'ils parviennent à mesurer plus précisément la taille du creusement de la tempête que les prévisionnistes prennent conscience du 
caractère exceptionnel de la menace et qu'ils tentent d'avertir, cette fois de manière beaucoup plus alarmiste la sécurité civile. Pourtant, la transmission orale de l'alerte dans le département étudié est influencée par les effets du contexte d'action situé. Le directeur départemental de MétéoFrance qui tente d'avertir le préfet illustre ce cas de figure. En effet, le représentant de l'État ne croit pas le météorologue qui tente pourtant d'attirer son attention sur le danger imminent que représente cette tempête. Mais pour comprendre cette attitude apparemment surprenante, il faut revenir au préalable sur un événement survenu peu avant, l'échec du plan Polmar (pollution marine) appliqué suite au naufrage de l'Erika.

Après le naufrage du tanker maltais l'Erika le 12 décembre 1999, le préfet est nommé responsable interdépartemental du plan Polmar. Une cellule de protection côtière interdépartementale est installée dans le département. Elle réunit l'ensemble des moyens de secours humains et matériels en charge des opérations de pompage des nappes de fioul en mer et sur terre. Les agents départementaux de Météo-France participent à ces opérations. Leur modèle «Mothy » simule les futurs déplacements des nappes d'hydrocarbures. Le 22 décembre, au cours d'une réunion quotidienne de la cellule de coordination, Météo-France confirme la tendance des prévisions établies les jours précédents : la plus grande partie des nappes de fioul atteindra les plages du département dans les prochaines échéances. Un militaire de la marine nationale, spécialiste de la navigation, contredit les prévisions les météorologues. D’après les simulations de la marine nationale, les nappes de pétrole vont s'échouer plus au nord, dans un périmètre situé entre la Loire-Atlantique et le Sud du Finistère. Le préfet semble atterré par cette déclaration : si les simulations de la marine sont exactes, il faudrait redéployer en toute urgence l'ensemble des moyens opérationnels vers la zone concernée. Mais cela entraînerait un important retard et les opérations de pompage devraient se faire directement sur terre et non plus sur mer, comme cela était initialement prévu. Le lendemain, le 23 décembre, les prévisions de la marine s'avèrent justes : les nappes de pétrole souillent les côtes de l'île d'Yeu et le Sud Finistère. Le même jour, et après lui avoir adressé une sérieuse remontrance, le ministère de l'Équipement retire la responsabilité du dispositif Polmar au préfet du département étudié au profit de son homologue de la Bretagne. L'ensemble du dispositif se rend en toute hâte à Rennes.

Dans la matinée du lundi 27 décembre, au cours d'une réunion à la préfecture, le préfet adresse publiquement un sévère réquisitoire à l'encontre de Météo-France. Le préfet tient Météo-France pour responsable de cet échec ${ }^{37}$ et somme le directeur départemental de lui fournir au plus vite une explication $^{38}$. La réunion terminée, aux alentours de 11 heures, le directeur départemental de Météo-France rentre à son bureau. Il trouve le BRAM 37.4 sur son bureau. Même s'il s'agit du troisième BRAM en deux jours, celui-ci lui apparaît plus préoccupant. Il est accompagné d'un bulletin ALARM mais plus important, il prévoit des forces de vents pouvant atteindre $150 \mathrm{~km} / \mathrm{h}$, un seuil peu courant. Il contacte ses collègues du CMIR qui lui font part des dernières informations alarmantes dont ils disposent sur la taille du creusement de la dépression. Même si sa trajectoire demeure encore incertaine, le CMIR conseille au directeur départemental de contacter personnellement le préfet pour l'avertir du caractère dangereux du phénomène. Mais il est accueilli

\footnotetext{
37 Météo-France et Mothy furent notamment impliqués pour «incompétence » par la commission d'enquête parlementaire mise en place mi-2000 suite au plan Polmar.

${ }^{38}$ Les prévisionnistes comprendront seulement plus tard l'origine de leur erreur. La reconnaissance aérienne effectuée par les avions des douanes qui a servi de base d'information au modèle Mothy n'a pu visualiser la majeure partie des nappes du fait des mauvaises conditions météorologiques. Un épais brouillard et des pluies persistantes n'ont pas permis un survol de plafond bas, qui aurait rendu possible la localisation des nappes. Sur la base de ces observations tronquées, Mothy a donc fourni logiquement de fausses prévisions.
} 
froidement par le préfet qui ne semble plus accorder un grand crédit à l'expertise des météorologues après l'épisode de Polmar. Toujours sous le coup de cet échec, le directeur départemental se trouve lui-même à court d'arguments. Il laisse le préfet interrompre sèchement la conversation.

Cet exemple témoigne de la fragilité de l'expertise institutionnelle de Météo-France. Celle-ci dépend non seulement de la qualité des relations interpersonnelles mais de plus, elle est soumise aux contingences du contexte d'action.

Ce niveau d'analyse permet de compléter les mécanismes qui agissent dans la prévision météorologique : la prévision à Météo-France est le produit d'un système sociotechnique où l'expertise humaine et les artefacts interagissent dans l'action au sein d'un environnement immédiat (Conein et Jacopin, 1994). La convergence des résultats des modèles structure le processus de décision de la prévision météorologique. Plus les résultats des différents modèles convergent et moins l'expertise humaine et les contraintes organisationnelles pèsent sur le choix du scénario de la prévision. La preuve mathématique, qui possède une force de conviction supérieure du fait de son caractère synoptique (Wittgenstein, 1983), lève les réticences des prévisionnistes, met fin aux débats et permet aux différents échelons de Météo-France de s'accorder. Inversement, plus les résultats des modèles diffèrent, et plus l'expertise humaine, soumise aux contingences contextuelles et aux contraintes organisationnelles prend une importance accrue ${ }^{39}$. Ce mécanisme qui peut apparaître trivial pour la sociologie des sciences, a des répercussions importantes pour la prévision des catastrophes naturelles. En effet, comme les phénomènes violents et anormaux aux conséquences dévastatrices échappent généralement aux modèles numériques, on peut conclure que leur prévision par Météo-France est soumise, plus que toute autre, aux contingences du contexte d'action.

\subsection{La réception de l'alerte: une menace familière}

Pour saisir le poids des effets contextuels qui ont pesé sur l'interprétation de cette alerte, nous distinguerons ceux du long terme (les précédents épisodes de tempête dans le département) et du court terme (le contexte du mois de décembre 1999).

Sur le long terme, dans le département côtier étudié, les vents violents d'hiver pouvant dépasser les $100 \mathrm{~km} / \mathrm{h}$ sont très fréquents. Cette accoutumance aux tempêtes d'hiver contribue à dénaturer la menace représentée par les avis de vigilance diffusés par Météo-France. Cela renforce la tendance à ne pas s'inquiéter et donc les réactions routinières quelles soient passives ou, au contraire, réactives face à ces bulletins. Ainsi, rompus aux tempêtes d'hiver en cette période, les marinspêcheurs hésitent d'autant moins à prendre la mer ce jour-là. À l'inverse, c'est aussi parce qu'ils se sont habitués à subir des vents violents que la SNCF ou encore les autorités départementales ne s'inquiètent pas outre mesure lorsqu'ils reçoivent les BRAM du 27 décembre.

Sur le court terme, c'est la répétition de fausses alertes à la tempête qui a précédée celle du 27 décembre qui contribue à une interprétation routinière du signal en brouillant la lecture du seuil de $150 \mathrm{~km} / \mathrm{h}$ annoncé comme exceptionnel: du 25 au 26 décembre, Météo-France diffuse une série d'avis de tempête sans que cela occasionne de dégâts notables dans le département.

\footnotetext{
39 Pour généraliser un peu plus notre propos, on peut se référer au travail de Juan Manuel Corvalan. Dans l'exemple des inondations du Gard des 8 et 9 septembre 2002, l'auteur montre comment un chef prévisionniste a choisi d'alerter, de sa propre initiative et, contre l'avis de sa hiérarchie, la sécurité civile de la dangerosité de l'orage qui menaçait la région. Ce prévisionniste s'appuyait, en effet, sur les résultats d'un modèle américain, alors que Météo-France hésitait à lancer une alerte rouge sur la base des résultats d'un modèle autre qu'ARPEGE, toujours pour satisfaire son obligation de moyens (Corvalan, 2005).
} 
Ainsi, lorsque les agents du service de la sécurité civile (SCIACPC) de la préfecture par exemple, reçoivent les BRAM et ALARM du 27 décembre, ils ne voient pas réellement de raison de s'inquiéter. Depuis quelques jours, Météo-France les met en garde sur le passage de plusieurs dépressions susceptibles d'occasionner des dégâts. Or, si les vents de ces derniers jours ont bien entraîné quelques dommages mineurs (chutes de branches, tuiles détruites, etc.) ainsi qu'une hausse de l'activité des sapeurs-pompiers, il ne s'est rien produit de vraiment alarmant. Pour le SCIACPC, les BRAM des 25 et 26 décembre sont donc interprétés comme des «fausses alertes » puisque les vents n'ont pas entraîné de dégâts notables. Cette attitude est, du reste, confortée par les doutes que les agents expriment vis-à-vis de la fiabilité des bulletins d'alerte météorologiques. D'après eux, généralement, seulement la moitié des bulletins donne lieu à des perturbations ${ }^{40}$. Mais inversement, ces précédents du temps court contribuent à renforcer la vigilance des acteurs ayant appliqué des mesures préventives. Ainsi, depuis plusieurs semaines, les météorologues conseillent vivement aux exploitants pêcheurs de limiter leurs sorties en mer en raison d'un contexte très perturbé sur l'Atlantique, propice à la formation des tempêtes. Du reste, en cette période de Noël, l'activité de la pêche est considérablement ralentie dans le département ${ }^{41}$.

Plus généralement, lorsqu'ils prennent connaissance des bulletins d'alerte du 27, les acteurs font l'amalgame entre la puissance des vents hivernaux habituels, les valeurs annoncées dans les précédents BRAM et celles des bulletins BRAM et ALARM du 27 décembre 1999.

«Je reste persuadé qu'avec ce type de télex on ne pouvait rien soupçonner. [...] Des avis de tempêtes où il n'y a pas eu de tempête on en a eu des tonnes, on s'était souvent fait avoir sur des télex qui n'ont rien donné, à la louche je dirais que ça correspondait à peu près à $60 \%$ des cas. Le problème aussi, c'est que l'on était en BRAM depuis quelques jours et même s'il est vrai que ça avait un peu soufflé fort, on n'avait rien recensé de désastreux. Alors c'est vrai, on a eu du mal à y croire. » SIACPC Préfecture.

Enfin, on retrouve une nouvelle fois, l'influence de la courte échéance à laquelle est donnée l'alerte par Météo-France sur l'entreprise de normalisation du signal. Comme précisé précédemment, lorsqu'ils réalisent que la tempête prend des proportions catastrophiques, en début d'après-midi, les personnels de Météo-France n'ont pas le temps de prévenir l'ensemble des acteurs du réseau de sécurité civile. Mais de manière plus fondamentale, un délai aussi court nuit directement à la possibilité de croire à un scénario catastrophe. Durant les quelques heures qui séparent les premières diffusions des bulletins et les premiers effets de la tempête, les acteurs ont peu de temps pour prendre conscience du danger. On imagine, en effet, qu'avec plus de recul et surtout avec d'autres sources d'information que celles Météo-France, les acteurs de la sécurité civile auraient été plus à même de fonder le caractère tangible (Chateauraynaud et Torny, 1999) de la menace. Ainsi, le préfet, qui a été prévenu dans la matinée de l'arrivée de la tempête, aurait certainement pu mieux apprécier la menace, s'il avait pu recouper plusieurs sources d'informations et ne pas dépendre seulement de Météo-France.

En conséquence, ce niveau d'analyse permet d'établir que l'annonce de la tempête est en quelque sorte diluée par le contexte d'action dans lequel elle intervient. Tout d'abord, le contexte de l'alerte manquée de Lothar contribue à atténuer la menace dans la transmission orale de l'alerte

\footnotetext{
${ }^{40} \mathrm{Ce}$ constat ne s'applique pas au cas unique du département étudié. Nous nous référons encore une fois à l'étude de J. Corvalan (2005). Dans sa monographie, l'auteur montre que c'est au niveau préfectoral que la fiabilité des avis de vigilance météorologique est la plus critiquée.

41 Seuls quelques petits exploitants continuent de pêcher mais dans un périmètre restreint, généralement, aux alentours des côtes du département.
} 
par les météorologues. Ensuite, l'accoutumance des acteurs locaux de la sécurité civile aux vents violents d'hiver et la répétition des fausses alertes diffusées par Météo-France les jours précédents la tempête brouillent la lecture des seuils présentés comme alarmant et dénature donc la menace. Enfin, le délai court parachève l'interprétation routinière du signal puisque non seulement certains acteurs comme les mairies sont prévenues trop tard mais, surtout, les acteurs n'ont en quelque sorte «pas le temps » de prendre conscience de la nature exceptionnelle de la menace en ayant la possibilité de recouper des informations. En conséquence et malgré les diffusions répétées écrites et orales de l'alerte météorologique, les acteurs ne s'attendent pas à l'arrivée d'une catastrophe et vaquent à leurs activités routinières sans conscience de la catastrophe qui se prépare.

\section{Les raisons interactionnistes : comment croire les prophètes de malheur?}

Nous allons à présent réduire l'échelle de l'observation du système d'alerte météorologique, pour nous intéresser aux individus qui l'animent. Le 27 décembre, dans le vaste réseau d'organisations impliquées dans la sécurité civile, tandis que la plupart poursuivent leurs activités routinières, certains individus s'alarment de la tempête qui approche. Voyons, d'abord, comment ces acteurs parviennent à déceler les signaux précurseurs de la catastrophe (les sources de l'alarme) et ensuite, dans quelle mesure ils parviennent à alerter leur entourage. La transmission de ces alertes dans les interactions de face-à-face ne va pas de soi puisqu'elles sont lancées de manière improvisée, en dehors de toutes mesures préventives prédéfinies. Pour comprendre pourquoi ces alertes sont entendues ou non, nous allons nous appuyer sur deux cas: celui d'un sous-préfet qui tente d'avertir les maires de son arrondissement et d'un ostréiculteur alertant les gendarmes de sa commune.

\subsection{Les sources cognitives de l'alarme}

Au niveau individuel, les sources de l'alarme des lanceurs d'alerte sont cognitives ${ }^{42}$. Il s'agit d'une activité de mise en relation qui s'opère entre un signal et une connaissance issue soit d'une expérience passée soit d'une expertise empirique particulière. Ainsi, le sous-préfet s'alerte lorsqu'il prend connaissance de la vitesse des vents annoncée dans le premier BRAM (37.4). Cet ancien gradé militaire qui a exercé ses fonctions à Nouméa a subit plusieurs tempêtes et connaît les différents seuils de l'échelle de Saffir-Simpson ainsi que les destructions qui y sont associés. Il anticipe alors les dommages que des vents de $150 \mathrm{~km} / \mathrm{h}$ vont occasionner sur les forêts. C'est bien ce qui le préoccupe. Dans son arrondissement, un grand nombre de communes sont situées à proximité de la grande forêt du département qui couvre environ 30000 hectares. Dans l'après-midi, il contacte le service départemental de Météo-France pour connaître les dernières évolutions de la tempête. Le météorologue confirme ses craintes : les vitesses de vents seront très certainement supérieures à celles annoncées dans la matinée. Il ajoute que la tempête se dirige tout droit vers le sud-ouest de la France. Ainsi, alors que nous avons vu que la plupart des acteurs ont fait l'amalgame entre la vitesse des vents des tempêtes d'hiver habituelles et celle annoncée le 27 décembre par Météo-France, l'expérience personnelle de cet acteur lui permet de différencier ces différents seuils.

Pour un ostréiculteur d'une petite commune côtière du sud du département, le sentiment d'alarme naît d'une connexion entre son expertise empirique et l'observation de l'environnement

\footnotetext{
42 Nous utiliserons le terme cognitif au sens où l'emploie Erhard Friedber, à savoir «une activité de perception, de compréhension, d'évaluation et aussi d'anticipation intelligente» (Friedberg, 1993, p. 208).
} 
géographique immédiat. Le lundi 27 décembre, deux gendarmes de Port-des-Barques patrouillent dans une petite île faisant face à la commune. Ils sont chargés de constater si les rafales de vent de la veille ont occasionné des dégâts sur l'île. En chemin, les gendarmes rencontrent cet ostréiculteur. Ce dernier attire leur attention sur le fait que la marée monte à une vitesse anormale depuis une heure : il est à peu près $16 \mathrm{~h} 30$ et la hauteur de la mer a atteint son coefficient maximal de marée, initialement prévu à 20 heures. Il voit là une chute de pression particulièrement brutale. L'ostréiculteur effraie donc littéralement les gendarmes quand il leur certifie que la mer va passer par-dessus les digues du front de mer dans les prochaines heures, pour submerger les habitations et les commerces du front de mer. Il se montre catégorique puisqu'il a vécu un phénomène similaire, il y a une vingtaine d'années et que cela avait entraîné l'inondation de la partie basse de la ville.

Les sources de l'alerte sont liées à des expertises individuelles associées aux tempêtes que Brian Wynne décrit comme un «lay knowledge» (Wynne, 1996). Ces connaissances suscitent le sentiment d'alerte parce qu'elles permettent d'établir un lien de causalité entre un signal (de nature diverse) et les dégâts attendus. Cette activité de mise en relation cognitive déjà décrite par Pierre Bourdieu comme un commutateur (Bourdieu, 1980) permet de transformer un «passif» en un principe «actif». Autrement dit, elle permet de créer du lien entre le passé et le présent, l'un donnant sens à l'autre.

\subsection{La transmission de l'alerte de face-à-face : l'expertise subjective à l'épreuve}

Une fois alarmés, ces acteurs tentent d'alerter leur entourage. Mais cela est problématique puisque, à l'instar des lanceurs d'alerte décrits par Francis Chateauraynaud et Didier Torny, ces individus n'ont pas de légitimité officielle ${ }^{43}$ pour émettre cette alerte. De surcroît, ils alertent généralement leur entourage selon des modes inhabituels et, la plupart du temps, improvisés.

Comme l'attestent nos deux exemples, en situation de face-à-face, l'alerte est entendue lorsque l'expertise subjective du lanceur d'alerte a été préalablement éprouvée. Ainsi, si les deux gendarmes croient immédiatement l'ostréiculteur c'est parce qu'ils accordent le plus grand crédit à son expertise sur le milieu marin. En effet, la brigade fait régulièrement appel à lui lorsqu'ils ont besoin d'informations sur l'état de la mer et des marées. Aussi, et après les avertissements de l'ostréiculteur, les gendarmes retournent en toute hâte dans la commune pour prévenir la population. Les deux militaires parent au plus pressé : ils préviennent les habitants du bord de mer et les élus présents à la mairie de la catastrophe à venir. Ils se livrent ensuite à un rapide porte-à-porte dans les commerces et les résidences situées au plus près du front de mer. «Fermez vos maisons, déplacez vos voitures du parking proche de la plage et mettez-vous à l'abri parce que la mer va passer par-dessus » conseillent-ils aux personnes qu'ils rencontrent. Les voitures sont déplacées et les commerces du front de mer ferment immédiatement.

Le cas du sous-préfet constitue le pendant inverse puisque sa tentative d'alerte envers les maires de son arrondissement échoue. Lorsque Météo-France lui confirme la prégnance de la menace, le sous-préfet et ses collaborateurs téléphonent, vers $17 \mathrm{~h} 30$, aux communes situées aux alentours de la forêt pour qu'ils se prémunissent contre les chutes d'arbres. Ses avertissements ont pourtant

\footnotetext{
43 Nous employons ce terme à dessein. En effet, ces lanceurs d'alerte possèdent des caractéristiques similaires à ceux décrits par F. Chateauraynaud et D. Torny. Pour les auteurs, il s'agit «de personnages non officiels qui bénéficient d'une faible légitimité provenant de personnes d'instances autorisées mais qui lancent des alertes individuelles selon des procédures habituelles [...] ici le lanceur d'alerte n'est pas un expert» (Chateauraynaud et Torny, 1999, p. 14). De même, nos lanceurs d'alerte sont des acteurs qui n'ont pas de légitimité officielle ou institutionnelle particulière lorsqu'ils cherchent à prévenir leur entourage.
} 
peu d'écho parmi les rares communes qu'ils parviennent à joindre ${ }^{44}$. Le sous-préfet a l'impression qu'il n'est pas vraiment pris au sérieux par ses interlocuteurs. Le secrétaire général d'une petite commune $^{45}$, notamment, lui avoue qu'il a du mal à croire qu'une tempête puisse occasionner de tels dégâts. Il se permet de lui préciser que son expérience à Nouméa lui est mauvaise conseillère.

Ainsi, lorsqu'ils cherchent à leur tour à avertir leur entourage, c'est la crédibilité éprouvée de l'expertise subjective du lanceur d'alerte sur ce domaine qui permet à ces alertes d'être entendues. Les conditions qui fondent cette crédibilité sont préexistantes à la situation et dépendent de la manière dont l'expertise du lanceur d'alerte a été éprouvée au préalable.

En conséquence, ce troisième niveau d'analyse montre que seuls quelques individus, forts de leurs expériences passées ou de leur expertise, parviennent à anticiper la catastrophe. Pourtant, la portée de ces alertes de face-à-face reste globalement limitée dans l'épisode de 1999 comme nous avons pu le voir à travers ces exemples ainsi que celui de la tentative d'alerte du directeur départemental de Météo-France envers le préfet, évoquée plus haut. Ces alertes ne permettent pas de rompre sensiblement avec les routines organisationnelles qui restent le mécanisme dominant dans l'exercice de la vigilance au cours de cet épisode. Mais ces différents exemples permettent de souligner la précarité de l'identification de signaux moins ordinaires : cela dépend, d'une part, de la présence d'individus aux savoirs particuliers et, d'autre part, des conditions de transmission de l'alerte dans les interactions de face-à-face.

\section{Conclusion}

L'effet de surprise de la catastrophe de 1999 ne peut être associé à un dysfonctionnement de l'alerte météorologique dont la procédure a été, au contraire, respectée à la lettre. Si l'alerte a été ignorée, c'est en raison de l'apparence familière du phénomène. L'épisode de 1999 constitue une catégorie de risque (Borraz, 2008) ${ }^{46}$ particulier que nous qualifierons de «scélérat ». À l'instar des entreprises scélérates étudiées par Erving Goffman (1973), le risque scélérat se définit, d'une manière générale, comme un phénomène menaçant dont l'annonce passe inaperçue, parce qu'il prend une apparence normale.

Le cas de la tempête de 1999 permet de dresser un idéal-type du risque scélérat. La propension de ce danger à devenir «scélérat » est une combinaison de deux caractéristiques. Première caractéristique, la qualité scélérate de ce risque tient, d'abord, aux caractéristiques substantielles de l'agent destructeur à la fois «hors cadre» et familier. Les tempêtes sont, en effet, des phénomènes connus et régulièrement éprouvés en France et particulièrement dans le territoire côtier étudié. Mais la puissance des vents de Martin, associée à son extension géographique, lui donne un caractère extraordinaire. Le caractère exceptionnel de l'événement n'est donc pas lié à sa nature mais plutôt à son ampleur. C'est pourquoi ce type de risque dépasse les capacités de prévention ordinaires puisque nous avons pu montrer qu'il n'existait pas réellement de mesures préventives adaptées à ce type de risque. Cette caractéristique substantielle à la fois

\footnotetext{
44 Comme cela était précisé plus haut, un grand nombre de communes sont fermées en cette fin d'après midi.

45 Précisons ici que nous n'avons pas pu recouper l'information auprès de cet acteur qui n'était plus en poste au moment de notre enquête.

46 Nous employons le terme risque, généralement employé de manière imprécise par la littérature (Alexander, 1993), au sens premier du terme, à savoir un danger inhérent à une activité ou, plus précisément, une opération de classification de ce qui est dangereux ou pas dans un système organisé donné. Selon la définition qu'en donne Olivier Borraz, ces opérations de classification obéissent à un processus dynamique mêlant des systèmes techniques de mesure, des procédures et des agents collectifs et individuels évoluant dans un contexte donné (Borraz, 2008).
} 
«hors cadre» et «familière » confère au phénomène un potentiel «scélérat». Mais, et c'est là sa seconde caractéristique, ce qui fonde la familiarité apparente du danger est un construit social qui se manifeste, dans notre cas, par la résonance ${ }^{47}$ du phénomène avec le système interorganisationnel d'alerte météorologique. C'est, plus précisément, la résonance du phénomène à deux niveaux, qui lui permet de passer inaperçu aux yeux des acteurs chargés d'interpréter l'alerte. Tout d'abord, sa résonance dans le système technique de Météo-France lui permet d'être repéré seulement dans un court terme. Le délai court auquel est donné l'alerte participe à l'entreprise de normalisation du signal parce qu'il réduit les possibilités de recouper les informations qui pourraient permettre de prendre conscience de la nature exceptionnelle de la menace. Ensuite, ce sont les particularités du contexte d'action situé et, notamment la répétition de fausses alertes, qui ont dénaturé le caractère exceptionnel de la menace aux yeux des différents récepteurs.

La notion de risque scélérat se distingue des thèses de la déficience qui défendent l'idée que «l'on aurait pu savoir». En ayant le souci de reconstruire ex ante les logiques de la diffusion et de la réception de l'alerte, nous souhaitions éviter l'écueil de la reconstruction a posteriori des responsabilités dans l'échec de la prévention de cette catastrophe. On pourrait certes relever dans notre analyse des séquences d'action qui pourraient pencher en faveur de la thèse de la déficience. On pourrait, par exemple, considérer que les météorologues du CMIR ont fait preuve de négligence lorsque, la veille du passage de la tempête, le SCEM leur a recommandé d'avertir les autorités. Mais, outre le fait que l'argument d'une défaillance est discutable sur le plan organisationnel et juridique ${ }^{48}$, raisonner de cette manière, c'est-à-dire par une reconstruction ex post des faits, a tendance à gommer les contraintes qui pèsent sur les acteurs dans la situation d'alerte. En l'occurrence ici, la prudence des prévisionnistes s'explique par l'anticipation d'une évaluation négative interne de leur prévision due, à ce moment-là, à la pression de l'échec de la prévision de la tempête de la vieille et d'une répétition trop rapprochée de fausses alertes. Mais, plus important, nous pensons qu'il est plus fécond de considérer cette attitude, non pas comme un fait isolé mais plutôt comme le rouage d'un enchaînement causal plus vaste qui permet d'expliquer pourquoi l'alerte est entendue ou non. Ainsi, la prudence des CMIR a eu pour conséquence majeure de retarder la diffusion de l'alerte, ce qui, ensuite, a contribué à restreindre les possibilités d'action préventive de la sécurité civile mais a aussi réduit les possibilités de croire à la possibilité d'une catastrophe. C'est donc bien dans une dynamique globale composée de plusieurs actions autonomes qui ont des répercussions les unes sur les autres, que cette alerte s'est transformée, progressivement et successivement dans la chaîne d'alerte, en un signal normal.

En somme, le risque scélérat, élaboré à partir d'une reconstruction ex ante et dynamique de la situation d'alerte, permet de soutenir l'idée qu'il était difficile de savoir, même dans le cas d'une alerte donnée explicitement comme ce fut le cas en 1999. Cela implique que le risque scélérat induit un implicite peu rassurant par rapports aux thèses de la défaillance des systèmes d'alerte. En effet, cette thèse en pointant des dysfonctionnements, postule de manière implicite qu'il existe toujours des voies d'amélioration possible pour prévenir les catastrophes. Or, dans le cas des risques scélérats et comme on a pu le voir à travers ce cas, l'alerte a bien été donnée et

\footnotetext{
47 Nous empruntons le terme «résonance» aux sciences physiques qui le définit de manière générique comme «l'augmentation de l'amplitude d'oscillation d'un système physique lorsque celui-ci est excité au voisinage d'une de ses fréquences proches ». Définition proposée par le dictionnaire Trésor de la langue française informatisée (TLFI).

48 On peut considérer, en effet, que le CMIR, même s'il s'est montré prudent, a bien donné l'alerte, une fois qu'il disposait de l'ensemble des moyens pour le faire, c'est-à-dire la convergence des modèles. Il a de cette manière satisfait à son obligation de moyen qui reste, rappelons-le, la principale source d'évaluation de Météo-France.
} 
les acteurs se sont bien montrés vigilants. Du fait de son apparence normale, l'identification des signaux précurseurs d'un danger n'est pas à chercher dans les signaux faibles mais bien dans les signaux normaux. La prévention d'un risque scélérat impliquerait donc de voir un danger derrière les signaux normaux, ce qui ne peut que conduire les agents en charge de la surveillance à la paranoïa et, à terme, à la paralysie de l'action.

Ces résultats nous amènent également à souligner la spécificité des risques naturels dans l'analyse des catastrophes et des accidents. Les perspectives d'analyses dites des vulnérabilités internes (Gilbert, 1992 ; Gilbert, 2005) prônent pour principe que les catastrophes naturelles ne seraient pas si «naturelles» puisqu'elles naissent non pas d'un «agent destructeur» mais plutôt au sein d'un contexte social qui, en fonction de ses caractéristiques, peut le transformer en désastre ou en simple incident. La sociologie américaine sur les désastres reprend cette question en s'opposant sur la place de l'agent physique dans l'analyse sociologique. Cette interrogation leur permet de débattre des différences existantes entre les catastrophes naturelles et technologiques (Kroll-Smith et al., 2005). Une partie de ces auteurs considèrent que les catastrophes naturelles occupent une place à part dans le champ des désastres en raison des particularités des phénomènes physiques qui sont à l'origine de ces situations. Inversement, d'autres, à l'instar du courant des «vulnérabilités internes », soutiennent qu'il n'y aurait aucune différence entre les désastres technologiques et naturels puisque leurs origines sont à rechercher au sein même des organisations ou des sociétés. Le cas exposé ici nous permet de nous situer dans ce débat. Contrairement au courant d'études des «vulnérabilités internes », nous pensons que l'analyse sociologique doit prendre en compte les particularités physiques de l'agent naturel étudié. Nous avons pu montrer qu'une partie des problématiques des alertes dans le cas des deux tempêtes de 1999, en premier lieu le délai auquel elles sont données, dépendent aussi des caractéristiques physiques du phénomène climatique : si l'alerte a été donnée en temps réel dans le cas de la tempête Lothar, c'est avant tout parce que cette tempête s'est formée à une vitesse exceptionnelle. Inversement, si MétéoFrance a pu donner l'alerte 12 heures avant son apparition sur la France, c'est d'abord parce que son stade de formation a été plus lent. Plus généralement, nous pensons que les mécanismes sociaux sont en partie influencés par des facteurs «extérieurs » et en l'occurrence ici environnementaux.

Cela nous amène à relever une dernière implication de la notion du risque scélérat. La méthodologie employée pour analyser ce cas permet de montrer que chaque catastrophe possède sa propre chaîne de causalité, ce qui implique, par conséquent, que les échecs de l'alerte annonçant les catastrophes sont à chaque fois spécifiques. Ce n'est pas contradictoire avec la généralisation de la notion de risque scélérat. On peut imaginer opérer, selon nous, des regroupements dans les causes conduisant à l'absence d'anticipation des catastrophes. C'est à ce titre que nous pensons que les risques scélérats forment une catégorie particulière d'explication à ce problème récurrent. Pour cela, il faudrait confronter soit l'idée défendue par cette notion (un danger annoncé comme tel mais ignoré parce qu'on s'y est accoutumé) soit l'idéal-type dressé à partir de ce risque naturel, à l'examen empirique d'autres situations de catastrophe ou de situations de surveillance plus ordinaires (contrôle aérien, surveillance épidémiologique ou des feux de forêt).

\section{Remerciements}

Nous tenons vivement à remercier François-Mathieu Poupeau (LATTS-CNRS), calembouriste alerte, pour ses patientes relectures et ses précieux conseils. 


\section{Références}

Alexander, D., 1993. "Natural Disaster”, UCL Press, London.

Baleste, M.-C., Brunet, H., Mougel, A., Coiffier, J., Bourdette, N., Bessemoulin, P., 2001. Les tempêtes exceptionnelles de Noël 1999. Phénomènes remarquables, vol. 7. Météo-France, Toulouse.

Bourdieu, P., 1980. Le sens pratique. Éditions de Minuit, Paris.

Borraz, O., 2008. Les politiques du risque. Presses de la Fondation nationale des sciences politiques, Paris.

Chateauraynaud, F., Torny, D., 1999. Les sombres précurseurs. Une sociologie pragmatique de l'alerte et du risque. Éditions de l'EHESS, Paris.

Conein, B., Jacopin, E., 1994. Action située et cognition : le savoir en place. Sociologie du travail 36 (4), $479-489$.

Corvalan, J., 2005. L'anticipation des catastrophes naturelles au moyen de la procédure de vigilance et d'alerte en France : alerte ou vigilance? Mémoire de Master IEP, Paris.

Desrosières, A., 2000. La politique des grands nombres : histoire de la raison statistique. La Découvete, Paris.

Francart, L., Dufour, I., 2002. Stratégie et décision, la crise du 11 septembre. La Découvete, Paris.

Frémaux, C., 2002. La responsabilité des maires face aux risques naturels. Les tempêtes: enseignement des crises et gestion des risques, réalités industrielles. Annales des Mines, 43-48.

Friedberg, E., 1993. Le pouvoir et la règle, dynamiques de l'action organisée. Le Seuil, Paris.

Gilbert, C., 2005. L'analyse des crises : entre normalisation et évitement. In: Gilbert, C., Borraz, O., Joly, P.-B. (Eds.), Risques, crises et incertitudes : pour une analyse critique. Cahiers du GIS risques collectifs et situations de crise, 3. CNRS-MSH-Alpes, Grenoble, pp. 175-223.

Gilbert C. 1992 «Le pouvoir en situation extrême. Catastrophe et politique ». L'harmattan, Paris.

Goffman, E., 1973. La mise en scène de la vie quotidienne, vol. 2. Éditions de Minuit, Paris.

Kroll-Smith, S., Couch, S., Levine, A.-G., 2005. Technological Hazards and Disasters. In: Dunlap, R.E., Michelson, W. (Eds.), Handbook of Environmental Sociology. Greenwood press, London, pp. 294-328.

Lagadec, P., Laroche, H., 2005. Retour sur les rapports d'enquête et d'expertise suite à la canicule de l'été 2003. In: Cahiers du GIS risques collectifs et situations de crise, vol. 4. CNRS-MSH-Alpes, Grenoble.

Ménard, C., 1997. L'économie des organisations. La Découverte, Paris.

Millet, M., 2005. Cadres de perception et luttes d'imputation dans la gestion de crise : l'exemple de «la canicule ». Revue française de sciences politiques 55 (4), 573-605.

Orléan, A., 2008. L'aveuglement au désastre : le cas des crises financières (entretien). Esprit, 9-19.

Polanyi, M., 1963. Experience and the Perception of Pattern. In: Sayre, K.M., Crosson, F.J. (Eds.), The Modeling of Mind, Computers and Intelligence. University of Notre Dame Press, Notre Dame, IN.

Sanson, G., Rochereau, O., Ravail, B., 2000. Mission interministérielle sur l'évaluation des dispositifs de secours et d'intervention mise en œuvre à l'occasion des tempêtes des 26 et 28 décembre 1999. Rapport pour le Premier ministre, Paris.

Vaughan, D., 1996. The Challenger Launch Decision. In: Risky Technology, Culture and Deviance at NASA. University of Chicago press, Chicago.

Vaughan, D., 2001. La normalisation de la déviance. In: Bourrier, M. (Ed.), Organiser la fiabilité. L’Harmattan, Paris, pp. 201-230.

Weick, K.E., 1995. Sensemaking in Organizations. Sage Publications, Thousand Oaks, CA.

Wittgenstein, L., 1983. Remarques sur le fondement des mathématiques. Gallimard, Paris.

Wynne, B., 1996. May the Sheep Safely Graze? A Reflexive View of the Expert Lay Knowledge Divide. In: Lash, S., Szerzynski, B., Wynne, B. (Eds.), Risk Environment and Modernity. Towards a New Ecology. Sage, London, pp. 44-83. 